\title{
Ärztliche Union für Kinder und Jugendliche
}

\section{$\mathrm{fPmh}$}

\section{Hélène Beutler}

Präsidentin fPmh

Korrespondenz:

Dr. med. Hélène Beutler

Kinder- und Jugendpsychiatrie

und -psychotherapie FMH

Leiterin UHPA

Beausite 47

CH-2740 Moutier

helene.beutler@gef.be.ch
In der Landschaft der Gesundheitspolitik stehen die Zeichen auf Veränderung, vor allem mit der Einführung von Tarifrevisionen (KVG, IV), der Entwicklung der Qualitätssicherung, der bevorstehenden Einführung von Managed Care sowie ab 2012 der DRG. In diesem Umfeld müssen sich die medizinischen Fachgesellschaften positionieren, eine qualitativ hochstehende Versorgung zu gewährleisten. Dies gilt ganz besonders für die medizinische Betreuung und Pflege von Kindern und Jugendlichen. Denn wie Erwachsene und ältere Menschen haben auch Kinder und Jugendliche Anspruch auf eine Betreuung und Pflege, die ihren Bedürfnissen entsprechen.

Ab 2003 haben sich die medizinischen Fachgesellschaften aus dem Bereich Kinder- und Jugendmedizin, die Schweizerische Gesellschaft für Pädiatrie (SGP), die Schweizerische Fachgesellschaft für Kinder- und Jugendpsychiatrie und -psychotherapie (SGKJPP) und die Schweizerische Gesellschaft für Kinderchirurgie (SGKC) zusammengeschlossen, um im November 2006 einen Dachverband zu gründen: Seither setzt sich die fPmh innerhalb der FMH für eine kindspezifische medizinische Betreuung ein.

Die spezifische medizinische Betreuung von Kindern und Jugendlichen beruht auf den folgenden Grundsätzen:

- Kinder haben Anrecht auf eine medizinische Betreuung von hoher Qualität, bei der ihre spezifischen Bedürfnisse berücksichtigt werden. Die Organisation der Betreuung, von der Diagnose bis zur Behandlung, hängt vom Alter und Entwicklungsstand jedes Kindes/Jugendlichen ab und trägt dem familiären, sozialen und schulischen Umfeld Rechnung.

- Damit eine auf die Bedürfnisse der Kinder abgestimmte Betreuung gewährleistet werden kann, muss ausgebildetes und qualifiziertes Personal zur Verfügung stehen. Deshalb ist in der Ausbildung der Fachpersonen, aber natürlich auch in der Weiterbildung der Ärztinnen und Ärzte und des paramedizi- nischen Personals vor allem den entwicklungsbezogenen Aspekten (auf somatischer, psychischer und zwischenmenschlicher Ebene) besondere Beachtung zu schenken.

- Die Gesundheitspolitik muss gewährleisten, dass Kinder in einem Rahmen und einer Infrastruktur betreut werden können, die auf ihre Bedürfnisse abgestimmt sind. Den Fachleuten muss sie ein vernetztes, interdisziplinäres Arbeiten ermöglichen, das über die Institutions- und Kantonsgrenzen hinaus anerkannt wird.

Ausgehend von diesen Grundprinzipien verfolgt die fPmh die folgenden Ziele:

- Aktives Engagement und Koordination im Gesundheitswesen, um gegenüber den Organen der FMH, den Fachgesellschaften, aber auch gegenüber santésuisse und den Gesundheitsdirektoren eine hochstehende Kinder- und Jugendmedizin zu fördern;

- Förderung der spezifischen wissenschaftlichen Forschung im Bereich der medizinischen Betreuung von Kindern und Jugendlichen;

- Ausbildung des beruflichen Nachwuchses;

- Aufbau von interdisziplinären Kompetenzzentren.

Die fPmh koordiniert ihre Tätigkeit über Arbeitsgruppen und Zusammenkünfte in den verschiedenen Gremien. Durch die regelmässige Organisation von gemeinsamen Kongressen der drei Fachgesellschaften fördert sie die interdisziplinäre Zusammenarbeit. Die erste derartige Veranstaltung fand 2008 zum Thema «Das Kind und sein Beziehungsnetz» statt. Für 2011 ist der nächste Kongress geplant, der sich den Fragen der Prävention widmen wird. In der Zwischenzeit werden in verschiedenen Regionen Kolloquien/Symposien organisiert, um die Bevölkerung für die aktuellen politischen Herausforderungen zu sensibilisieren, mit denen sich die Kinder- und Jugendmedizin konfrontiert sieht. 